\title{
Intra Operative Incidental Finding of a Proximal Jejunal Diverticulum:
} A Case Report

Amine Hamdane*, Omar Marghich, Hicham El Bouhaddouti, El Bachir Benjelloun, Abdelmalek Ousadden, Khalid Ait Taleb, Ouadii Mouaqit

Faculty of Medicine and Pharmacy, Sidi Mohammed Ben Abdellah University of Fez, Morocco, Visceral Surgery department A, CHU Hassan II, Fez, Morocco

DOI: $10.36347 /$ sasjs.2021.v07i05.005

| Received: 21.03.2021 | Accepted: 30.04.2021 | Published: 07.05.2021

*Corresponding author: Amine Hamdane

Abstract

Case Report

Diverticulosis of the small intestine in adults is rare [1], it is much less frequent in the jejunum than in the colon [2], the main complication of jejunal diverticulosis is perforation. We report the case of a 68 -year-old patient in whom a proximal jejunal diverticulum was discovered incidentally.

Keywords: Incidental Finding, Proximal Jejunal, Diverticulum.

Copyright $\left({ }_{0} 2021\right.$ The Author(s): This is an open-access article distributed under the terms of the Creative Commons Attribution 4.0 International License (CC BY-NC 4.0) which permits unrestricted use, distribution, and reproduction in any medium for non-commercial use provided the original author and source are credited.

\section{INTRODUCTION}

Diverticulosis of the jejunum is rare. Acquired diverticula result from dyskinesia and are drive diverticula [3-4]. In the absence of complications, small bowel diverticula are asymptomatic. However, the most common clinical manifestation is ill-defined abdominal pain, which is chronic in course [5]. Complications are often severe due to delayed diagnosis and thus management [1].

\section{PATIENT AND OBSERVATION}

A 68-year-old patient was admitted for a feeding jejunostomy. The patient has a history of an epidermoid carcinomaof the lower esophagus, candidate for concomitant radio-chemotherapy. The clinical examination found a malnourished and an aphagic patient with an altered general condition. Biological tests showed hypoalbuminemia at $20 \mathrm{mg} / \mathrm{l}$ with hypoprotidemia at $45 \mathrm{~g} / 1$.

The patient was admitted to the operating room, the surgical exploration had shown the presence of a jejunal diverticulum located $10 \mathrm{~cm}$ from the angle of Treitz ( Figure*), the decision was to respect the diverticulum, and to make a feeding jejunostomy according to Witzel downstream of the diverticulum. The postoperative course was uneventful. The patient was subsequently referred for radio chemotherapy sessions.

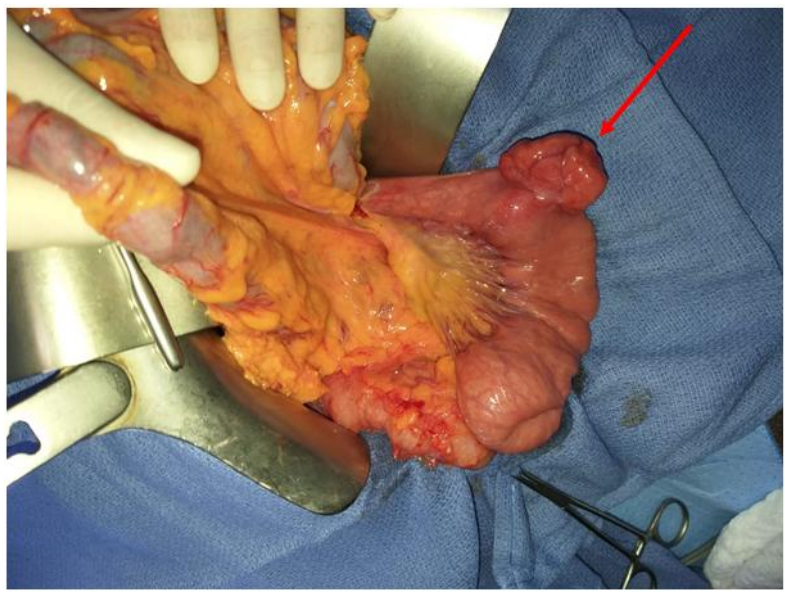

Fig-1: Proximal jejunal diverticulum

\section{DISCUSSION}

Acquired diverticula of the jejunum correspond to a herniation of the mucosa and submucosa and do not include muscularis, they are located on the weak areas of the jejunal wall, generally at the site of vessel penetration. Diverticulosis of the small intestine affects about $1 \%$ of the population, it is less frequent than that of the colon, the diverticula are multiple in two thirds of cases and predominate on the proximal jejunum, and their size is variable, sometimes $>10 \mathrm{~cm}$ [4-9]. More rarely, it may be a diverticulum of the distal ileum, often unique and of a small in size, and may pose a problem of differential diagnosis with Meckel's diverticulum [6]. 
Jejunaldiverticulums are found incidentally in $40 \%$ of cases. However, a malabsorption syndrome due to intraluminal microbial proliferation may occur. Other complications including perforation, occlusion and hemorrhage are observed in $10-20 \%$ of cases. Diverticulitis remains the rarest complication, it is of sub-acute evolution and presents as an inflammatory pseudotumor that evolves towards perforation with the risk of localized or generalized peritonitis [3-7].

The symptomatology of a complicated diverticulum is unspecific and misleading, and may simulate sigmoiditis, appendicitis or even cholecystitis $[6,7]$. Abdominal CT is the examination of choice for the positive diagnosis of a complicated bowel diverticulum [7-9], especially in terms of locating inflammatory phenomena on the mesentery and the possible presence of abscesses resulting from perforation, and to rule out other conditions such as sigmoiditis or appendicitis. The larger the diverticulum, the easier it is to detect, and the more aerated and heterogeneous the contents, the more likely it is to be related to intestinal stasis [1]. Diverticula collapsed by inflammation are not identifiable; in this case only inflammatory phenomena can be seen on the mesentery. Abdominal CT allows the extent of mesenteric infiltration to be determined, as well as the presence of radiological signs of severity such as abscesses, peritonitis and pneumoperitoneum, which indicate surgery.

Resection of the affected bowel segment with direct anastomosis is the treatment of choice [3-9], but this depends on local conditions. Some cases of diverticulitis have been treated medically depending on the terrain and especially in the absence of radiological signs of severity [6].

\section{CONCLUSION}

Small bowel diverticula are often discovered incidentally. However, they can be the cause of atypical abdominal pain with a chronic course or give a picture of peritonitis, occlusion or digestive hemorrhage. Abdominal CT is the reference examination for locating the diverticulum and diagnosing complications while eliminating other inflammatory conditions of the abdomen. Surgical resection with direct anastomosis of the diverticulum is the best option.

\section{RÉFÉRENCES}

1. Bassam Aboud, Rony Aouad, Joe Bou Jaoude, Claud Ghorra. A rare cause of actue abdomen: jejunal diverticulitis. La presse médicale. 2008; 37: 3.

2. Houssem Harbi, NizarKardoun, SamiFendri, NouhaDammak, NozhaToumi, Ahmed Guirat, Rafik Mzali. Jejunal diverticulitis. Review and treatment algorithm. La presse médicale 2017.

3. Longo WE, Vernava AR. Clinical implications of jejunoileal diverticular disease. Dis Colon Rectum. 1992; 35: 381-8.

4. Huguenin A, Tirveilliot F, Dell'erba U, Fabre S, Triboulet JP, Durand F. Les diverticules, jéjunoiléaux acquis (Meckel exclus). Ann Chir. 1999; 53: 522-6.

5. Cuningham SC, Gannon CJ, Napolitano LM. Small-bowel diverticulosis. Am J Surg. 2005; 190: 37-8?

6. Se' verin S, d'Alincourt A, Redon H, Hamy A, Mathon G, Lerat F. Diverticulite de l'intestin grêle: intérêt du scanner. Gastroenterol Clin Biol. 2003; 84:47-9.

7. Lin CY, Chang WH, Lin SC, Chu CH, Wang TE, Shih SC. muqueuse of clinical manifestationsof symptomatic acquired jejunoileal diverticulardisease. World J Gastroenterol. 2005; 11:5557-60.

8. Greenstein S, Jones B, FishmanEK, Cameron JL, Siegelman SS. Small-bowel diverticulitis: CTfindings. AJR. 1986; 147: 271-4.

9. Pans A, Taziaux P, Cobut M, Wahlen C. Ladiverticulose acquise de l'intestin grêle. JChir. 1992; 129:201-5. 